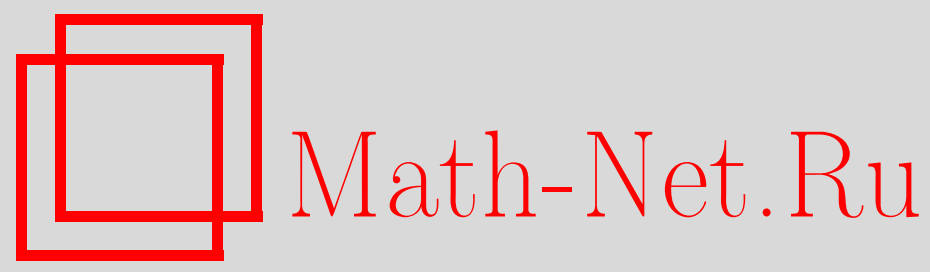

Л. П. Усольцев, О распределении последовательности дробных долей медленно растущей показательной функции, $M a$ тем. заметки, 1999, том 65, выпуск 1, 148-152

DOI: https://doi.org/10.4213/mzm1036

Использование Общероссийского математического портала Math-Net.Ru подразумевает, что вы прочитали и согласны с пользовательским соглашением http://www . mathnet.ru/rus/agreement

Параметры загрузки:

IP : 3.93 .64 .190

26 апреля 2023 г., 04:39:30

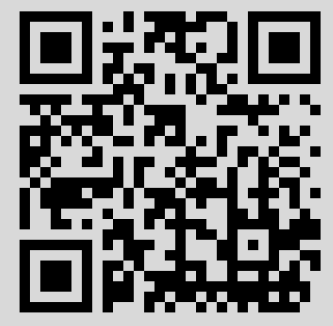




\section{О РАСПРЕДЕЛЕНИИ ПОСЛЕДОВАТЕЛЬНОСТИ ДРОБНЫХ ДОЛЕЙ МЕДЛЕННО РАСТУЩЕЙ ПОКАЗАТЕЛЬНОЙ ФУНКЦИИ}

\section{Л. П. Усольцев}

В работе рассматривается вопрос о степени отклонения распределения последовательности дробных долей медленно растущей показательной функции от "идеального" равномерного распределения. Дается оценка величины, которую уместно назвать мерой иррегулярности распределения данной последовательности.

Библиография: 2 названия.

В работе рассматривается вопрос о степени отклонения распределения последовательности дробных долей медленно растущей показательной функции от “идеального" равномерного распределения. Дается оценка величины, которую уместно назвать мерой иррегулярности распределения данной последовательности.

В метрической теории равномерного распределения дробных долей $\{\alpha f(n)\}, n=1$, $2, \ldots, N, N \rightarrow \infty$, сравнительно хорошо изучен случай $f(n)=q^{n}$, где $q>1$ - постоянная. А. Г. Постников привлек внимание автора к проблеме "вырождающегося" случая $q=q(N) \rightarrow 1$ при $N \rightarrow \infty$ (и, естественно, $\alpha=\alpha(N))$, когда традиционные методы исследования распределения дробных долей неприменимы. Предлагаемая работа является первым шагом в изучении данной проблемы. Поэтому предметно сравнивать полученные в работе результаты пока не с чем.

Пусть $\gamma>0$ - фиксированное вещественное число, $N$ - натуральная переменная, $q=e^{\gamma / N}$, а $\alpha=\alpha(N), \tau=\tau(N)$ и $l=l(N)$ - вещественные функции от $N$ такие, что $0<l \leqslant \tau N \leqslant \gamma^{2} e^{\gamma} \alpha<N$ при всех значениях $N$ и $l \gg \sqrt{\alpha \ln N}$ при $N \rightarrow \infty$.

Для любого подмножества $\Delta \subset[0,1)$ обозначаем через $Q_{\Delta}(N)$ количество натуральных чисел $n \leqslant N$, для которых $\left\{\alpha q^{n}\right\} \in \Delta$ и, как обычно, считаем, что

$$
\chi_{\Delta}(x)=\left\{\begin{array}{l}
1, \text { если }\{x\} \in \Delta, \\
0, \text { если }\{x\} \notin \Delta,
\end{array} \quad-\infty<x<\infty .\right.
$$

Таким образом,

$$
Q_{\Delta}(N)=\sum_{n=1}^{N} \chi_{\Delta}\left(\alpha q^{n}\right)
$$

Положим

$$
\Delta_{\tau}(\lambda)= \begin{cases}{[\lambda, \lambda+\tau),} & \text { если } 0 \leqslant \lambda \leqslant 1-\tau, \\ {[\lambda, 1) \cup[0, \lambda+\tau-1),} & \text { если } 1-\tau<\lambda<1,\end{cases}
$$

и докажем следующее утверждение. 
ТЕоРема. При $N \rightarrow \infty$ справедливо соотношение

$$
\operatorname{mes}\left\{\lambda \in[0,1):\left|Q_{\Delta_{\tau}(\lambda)}(N)-\tau N\right|>l\right\}=O\left(\frac{\alpha \ln N}{l^{2}}\right)
$$

с постоянной в знаке $O$, зависящей лишь от $\gamma$.

Считая $\tau$ фиксированньм числом из интервала $(0,1)$ и выбирая $\alpha=N$, получаем следующий (вероятно, наиболее интересньй) частный случай теоремы.

СледствиЕ. Пусть $\gamma$ и $\tau$ - фиксированные положительные вещественные числа, $0<\tau \leqslant \gamma^{2} e^{\gamma}<1 ; N$ - натуральная переменная, $\alpha=N, q=e^{\gamma / N} ; \psi(N)$ вещественная функиия от $N, 0<\psi(N) \leqslant \tau \sqrt{N / \ln N}$ и $\psi(N) \rightarrow \infty$ при $N \rightarrow \infty$. Тогда при $N \rightarrow \infty$ справедливо соотношение

$$
\operatorname{mes}\left\{\lambda \in[0,1):\left|Q_{\Delta_{\tau}(\lambda)}(N)-\tau N\right|>\sqrt{N \ln N} \cdot \psi(N)\right\}=O\left(\frac{1}{\psi^{2}(N)}\right) .
$$

ДоКАЗАТЕЛЬСТво ТЕОРЕМЫ. ВЫбирая произвольно натуральное число $N$, положим

$$
\begin{gathered}
R(\lambda)=Q_{\Delta_{\tau}(\{\lambda\})}(N)=\sum_{n=1}^{N} \chi_{\Delta_{\tau}(\{\lambda\})}\left(\alpha q^{n}\right), \quad-\infty<\lambda<\infty, \\
a_{m}=\int_{0}^{1} R(\lambda) e^{-2 \pi i m \lambda} d \lambda, \quad m \in \mathbb{Z} .
\end{gathered}
$$

Так как функция $R(\lambda)$ вещественнозначная, то

$$
a_{m}=\bar{a}_{-m}, \quad m \in \mathbb{Z} .
$$

Очевидно, при $0 \leqslant \lambda \leqslant 1-\tau$

$$
R(\lambda)=\sum_{n=1}^{N} \chi_{[\lambda, \lambda+\tau)}\left(\alpha q^{n}\right)=\sum_{n=1}^{N}\left(\chi_{\left(\left\{\alpha q^{n}\right\}, 1\right]}(\lambda+\tau)-\chi_{\left(\left\{\alpha q^{n}\right\}, 1\right]}(\lambda)\right),
$$

а при $1-\tau<\lambda<1$

$$
\begin{aligned}
R(\lambda) & =\sum_{n=1}^{N}\left(\chi_{[\lambda, 1)}\left(\alpha q^{n}\right)+\chi_{[0, \lambda+\tau-1)}\left(\alpha q^{n}\right)\right) \\
& =\sum_{n=1}^{N}\left(1-\chi_{\left(\left\{\alpha q^{n}\right\}, 1\right]}(\lambda)+\chi_{\left(\left\{\alpha q^{n}\right\}, 1\right]}(\lambda+\nu-1)\right) .
\end{aligned}
$$

Поэтому

$$
\begin{aligned}
a_{0}= & \int_{0}^{1} R(\lambda) d \lambda \\
= & \sum_{n=1}^{N}\left(\int_{0}^{1-\tau} \chi_{\left(\left\{\alpha q^{n}\right\}, 1\right]}(\lambda+\tau) d \lambda-\int_{0}^{1-\tau} \chi_{\left(\left\{\alpha q^{n}\right\}, 1\right]}(\lambda) d \lambda+\int_{1-\tau}^{1} d \lambda\right. \\
& \left.-\int_{1-\tau}^{1} \chi_{\left(\left\{\alpha q^{n}\right\}, 1\right]}(\lambda) d \lambda+\int_{1-\lambda}^{1} \chi_{\left(\left\{\alpha q^{n}\right\}, 1\right]}(\lambda+\nu-1) d \lambda\right)
\end{aligned}
$$




$$
\begin{aligned}
= & \sum_{n=1}^{N}\left(\int_{\tau}^{1} \chi_{\left(\left\{\alpha q^{n}\right\}, 1\right]}(\lambda) d \lambda-\int_{0}^{1-\tau} \chi_{\left(\left\{\alpha q^{n}\right\}, 1\right]}(\lambda) d \lambda+\tau\right. \\
& \left.-\int_{1-\tau}^{1} \chi_{\left(\left\{\alpha q^{n}\right\}, 1\right]}(\lambda) d \lambda+\int_{0}^{\tau} \chi_{\left(\left\{\alpha q^{n}\right\}, 1\right]}(\lambda) d \lambda\right) \\
= & \tau \sum_{n=1}^{N} 1=\tau N
\end{aligned}
$$

а при $m \neq 0$

$$
\begin{aligned}
& a_{m}=\int_{0}^{1} R(\lambda) e^{-2 \pi i m \lambda} d \lambda \\
& =\sum_{n=1}^{N}\left(\int_{0}^{1-\tau} \chi_{\left(\left\{\alpha q^{n}\right\}, 1\right]}(\lambda+\tau) e^{-2 \pi i m \lambda} d \lambda-\int_{0}^{1-\tau} \chi_{\left(\left\{\alpha q^{n}\right\}, 1\right]}(\lambda) e^{-2 \pi i m \lambda} d \lambda\right. \\
& +\int_{1-\tau}^{1} e^{-2 \pi i m \lambda} d \lambda-\int_{1-\tau}^{1} \chi_{\left(\left\{\alpha q^{n}\right\}, 1\right]}(\lambda) e^{-2 \pi i m \lambda} d \lambda \\
& \left.+\int_{1-\tau}^{1} \chi_{\left(\left\{\alpha q^{n}\right\}, 1\right]}(\lambda+\tau-1) e^{-2 \pi i m \lambda} d \lambda\right) \\
& =\sum_{n=1}^{N}\left(e^{2 \pi i m \tau} \int_{\tau}^{1} \chi_{\left(\left\{\alpha q^{n}\right\}, 1\right]}(\lambda) e^{-2 \pi i m \lambda} d \lambda-\int_{0}^{1-\tau} \chi_{\left(\left\{\alpha q^{n}\right\}, 1\right]}(\lambda) e^{-2 \pi i m \tau} d \lambda\right. \\
& +\frac{e^{2 \pi i m \tau}-1}{2 \pi i m}-\int_{1-\tau}^{1} \chi_{\left(\left\{\alpha q^{n}\right\}, 1\right]}(\lambda) e^{-2 \pi i m \lambda} d \lambda \\
& \left.+e^{2 \pi i m \tau} \int_{0}^{\tau} \chi_{\left(\left\{\alpha q^{n}\right\}, 1\right]}(\lambda) e^{-2 \pi i m \lambda} d \lambda\right) \\
& =\sum_{n=1}^{N}\left(e^{2 \pi i m \tau} \int_{0}^{1} \chi_{\left(\left\{\alpha q^{n}\right\}, 1\right]}(\lambda) e^{-2 \pi i m \lambda} d \lambda\right. \\
& \left.-\int_{0}^{1} \chi_{\left(\left\{\alpha q^{n}\right\}, 1\right]}(\lambda) e^{-2 \pi i m \lambda} d \lambda+\frac{e^{2 \pi i m \tau}-1}{2 \pi i m}\right) \\
& =\left(e^{2 \pi i m \tau}-1\right) \sum_{n=1}^{N}\left(\int_{0}^{1} \chi_{\left(\left\{\alpha q^{n}\right\}, 1\right]}(\lambda) e^{-2 \pi i m \lambda} d \lambda+\frac{1}{2 \pi i m}\right) \\
& =\left(e^{2 \pi i m \tau}-1\right) \sum_{n=1}^{N}\left(\int_{\left\{\alpha q^{n}\right\}}^{1} e^{-2 \pi i m \lambda} d \lambda+\frac{1}{2 \pi i m}\right) \\
& =\frac{e^{2 \pi i m \tau}-1}{2 \pi i m} \sum_{n=1}^{N} e^{-2 \pi i m \alpha q^{n}}=\frac{e^{2 \pi i m \tau}-1}{2 \pi i m} S_{-m},
\end{aligned}
$$

где

$$
S_{m}=\sum_{n=1}^{N} e^{2 \pi i m \alpha q^{n}}, \quad m= \pm 1, \pm 2, \ldots
$$


Используя последовательно равенства (2) и (4), неравенство Чебышева, равенство Парсеваля и равенства (3) и (5), получаем

$$
\begin{aligned}
& \operatorname{mes}\left\{\lambda \in[0,1):\left|Q_{\Delta_{\tau}(\lambda)}(N)-\tau N\right|>l\right\}=\operatorname{mes}\left\{\lambda \in[0,1):\left|R(\lambda)-a_{0}\right|>l\right\} \\
& \quad \leqslant \frac{1}{l^{2}} \int_{0}^{1}\left(R(\lambda)-a_{0}\right)^{2} d \lambda=\frac{1}{l^{2}} \sum_{m=-\infty}^{\infty}\left|a_{m}\right|^{2}=\frac{2}{l^{2}} \sum_{m=1}^{\infty}\left|a_{-m}\right|^{2} \\
& \quad \leqslant \frac{2}{l^{2}} \sum_{m=1}^{\infty}\left(\min \left(\tau, \frac{1}{\pi m}\right) \cdot\left|S_{m}\right|\right)^{2},
\end{aligned}
$$

где штрих у знака суммы означает, что из области суммирования исключено значение $m=0$.

Чтобы завершить доказательство теоремы, нам нужна оценка тригонометрической суммы $S_{m}$ с произвольным натуральным $m$.

Лемма. Существует абсолютная постоянная $B>0$ такая, что при всех натуральных т справедлива оченка

$$
\left|S_{m}\right| \leqslant B \min \left(N, \gamma e^{\gamma} \sqrt{m \alpha}+\frac{N}{\gamma \sqrt{m \alpha}}\right)
$$

ДоКАЗАТЕЛЬСТво. При всех натуральных $m$ положим

$$
f_{m}(y)=m \alpha q^{y}=m \alpha e^{\gamma y / N}, \quad 0 \leqslant y \leqslant N
$$

и запишем сумму $S_{m}$ в виде

$$
S_{m}=\sum_{n=1}^{N} e^{2 \pi i f_{m}(n)}
$$

На отрезке $0 \leqslant y \leqslant N$

$$
f_{m}^{\prime \prime}(y)=\frac{m \alpha \gamma^{2}}{N^{2}} e^{\gamma y / N} \leqslant \frac{m \alpha \gamma^{2}}{N^{2}} e^{\gamma} \quad \text { и } \quad f_{m}^{\prime \prime}(y) \geqslant \frac{m \alpha \gamma^{2}}{N^{2}}
$$

Но тогда в силу одной из классических лемм Ван дер Корпута (а именно, леммы, предваряющей метод Ван дер Корпута оценки тригонометрических сумм, см., например, [1, c. 107 , лемма 1] или [2, с. 27 , теорема 5]) существует абсолютная постоянная $B_{1}>0$ такая, что при всех натуральных $m$ справедлива оценка

$$
\left|S_{m}\right| \leqslant B_{1}\left(\gamma e^{\gamma} \sqrt{m \alpha}+\frac{N}{\gamma \sqrt{m \alpha}}\right) .
$$

Отсюда и из тривиальной оценки $\left|S_{m}\right| \leqslant N$ вытекает справедливость неравенства (7), например, с $B=B_{1}+1$. Лемма доказана. 
Завершим доказательство теоремы. Вследствии оценки (7) имеем

$$
\begin{aligned}
\sum_{m=1}^{\infty} & \left(\min \left(\tau, \frac{1}{\pi m}\right) \cdot\left|S_{m}\right|\right)^{2} \leqslant \sum_{m=1}^{\infty}\left(\min \left(\frac{\gamma e^{\gamma} \alpha}{N}, \frac{1}{\pi m}\right) \cdot\left|S_{m}\right|\right)^{2} \\
\leqslant & B^{2} \sum_{m \leqslant N /\left(\gamma^{2} e^{\gamma} \alpha\right)}\left(\frac{\gamma^{2} e^{\gamma} \alpha}{N} \cdot \frac{2 N}{\gamma \sqrt{m \alpha}}\right)^{2} \\
& +B^{2} \sum_{N /\left(\gamma^{2} e^{\gamma} \alpha\right)<m \leqslant N^{2}}\left(\frac{1}{\pi m} \cdot 2 \gamma e^{\gamma} \sqrt{m \alpha}\right)^{2}+B^{2} \sum_{m>N^{2}}\left(\frac{1}{\pi m} \cdot N\right)^{2} \\
= & 4 B^{2} \gamma^{2} e^{2 \gamma} \alpha \sum_{m \leqslant N /\left(\gamma^{2} e^{\gamma} \alpha\right)} \frac{1}{m} \\
& +\frac{4}{\pi^{2}} B^{2} \gamma^{2} e^{2 \gamma} \alpha \sum_{N /\left(\gamma^{2} e^{\gamma} \alpha\right)<m \leqslant N^{2}} \frac{1}{m}+\frac{1}{\pi^{2}} B^{2} N^{2} \sum_{m>N^{2}} \frac{1}{m^{2}} \\
\leqslant & 4 B^{2} \gamma^{2} e^{2 \gamma} \alpha \sum_{m \leqslant N^{2}} \frac{1}{m}+\frac{B^{2} N^{2}}{\pi^{2}} \cdot \frac{1}{N^{2}-1} \\
< & 4 B^{2} \gamma^{2} e^{2 \gamma} \alpha(1+2 \ln N)+B^{2}=O(\alpha \ln N)
\end{aligned}
$$

при $N \rightarrow \infty$. Отсюда и из (6) вытекает справедливость соотношения (1). Теорема доказана.

\section{СПИСОК ЦИТИРОВАННОЙ ЛИТЕРАТУРЫ}

[1] Титчмарш Е. К. Теория дзета-функции Римана. М.: ИЛ, 1953.

[2] Карацуба А. А. Основы аналитической теории чисел. М.: Наука, 1983.

Самарский государственный аэрокосмический университет 